\title{
Analysis of Field Errors in Existing Undulators
}

LBL $-282 B 8$

DE90 011614

BRIAN M. Kincaid

Levorence Berkeley Leboratory

Berkeley, CA 9/720

\begin{abstract}
The Advanced Light Source (ALS) and other third generation synchrotron light sources have been designed for optimum performance with undulator insertion devices. The performance requirements for these new undulators are explored, with emphasis on the effects of errors on source spectral brightness. Analysis of magnetic field data for several existing hybrid undulators is presented, decomposing errors into systematic and random components. An attempt is made to identify the sources of these errors, and recommendations are made for designing future insertion devices.
\end{abstract}

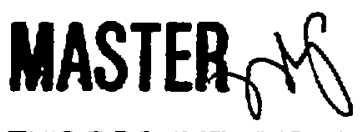

DISTFIBUTION OF THIS DOCUMENT IS UNLIMITED 


\section{Introduction}

The Advanced Light Source and (ALS) and other third generation synchrotron light sources have been designed for optimum performance with undulator insertion devices. The combined expectations of high spectral brightness, good harmonic output, stable and repeatable source size and position, and ease of tunability over a wide range of photon energies place a number of stringent requirements on the performance of this new generation of undulator insertion devices. Although the discussion in this paper focuses on storage ring synchrotron radiation sources and permanent magnet undulators, many of the conclusions also apply to other undulator-based radiation sources, such as the free electron laser. These requirements translate into tolerances for various kinds of field errors in the devices. In an attempt to understand the origin of errors in real undulators, an analysis of magnetic field data for several existing hybrid undulators has been performed, decomposing errors into systematic and random components. This analysis reveals that the dominant field error in these undulators is probably due to the non-ideal properties of permanent magnet blocks, for example, non-uniformity of magnetization in both magnitude and direction. The data analysis also reveals some of the desirable features of the Halbach hybrid unduiator design[1] and leads to suggestions for improving the design of future insertion devices.

\section{Efrect: of undulator field errors}

Undulator field errors can be classified as either random or systematic. Random errors are generally any deviation from a perfectly periodic undulator field and are the result of non-uniformities in magnetic materials and manufacturing tolerances, and can have a variety of deleterious effects on undulator performance. Systematic errors can take the form of spatial harmonics in the undulator field, integrated field errors due to improper magnetic design, ambient fields, etc. Both errors can produce unwanted deflection or focussing of the electron beam in an undulator, leading to undesirable beam motion and changes in the electron optics of the storage ring. They can also cause a reduction in spectral brightness of the output synchrotron radiation beam due to distortion of the periodic electron trajectory in the undulator. An example of this effect is shown in Fig. 1 . The calculated on axis irradiance spectrum 
(power per unit solid angle and frequency), shown in Fig. 2, shows a considerable drop in peak height at the $5^{\text {th }}$ harmonic. (See Ref. [2] for details).

One complication of the separation into random and systematic errors is that random errors may produce integrated field errors that are hard to distinguish from systematic errors, as will be seen in a later section. Another important criterion in the analysis of field errors is that the loss in performance depends strongly on the spatial distribution of the random field errorm with err.s hat produce beam deflection or

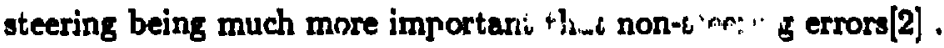

The acceptable level of random and systematic errors in a given insertion device depends on the spectral performance required and the properties of the electron beam and the storage ring. As an example, the proposed ALS U5.0 undulator, a 4.5 meter long, $5.0 \mathrm{~cm}$ period device with 89 full periods, is expected to produce useful radiation at the $5^{\text {th }}$ harmonic. Using Ref. [2], this leads to a RMS error tolerance specification of 0.25 percent. In addition, the requirement that the electron beam not be displaced by systematic integrated field errors leads to tolerances on integrated dipole and quadrupole fields of $100 \mathrm{G} \mathrm{cm}$ and $25 \mathrm{G}$, respectively[3]. This level of performance is beyond the present state of the art for permanent magnet undulators. It is therefore important to assess field errors in existing devices to identify the most important tolerances to be controlled.

\section{Analysis of existing devices}

Magnetic field data for three different insertion devices were analyzed, the LBLSSRL-EXXON beamline VI wiggler (BL VI)[4] the NSLS TOK undulator (TOK)[5] and the LLNL-LBL-SSRL beamline $X$ wiggler (BL $X)[6]$. The BL VI magnet is a samariam-cobalt/vanadium Permendur hybrid wiggler, the first such device built, instalied in 1983. Even though intended as a wiggler for use with a relatively large emittance electron beam, where the effects of field errors are less severe, the device has RMS random errors of 0.5 percent. The TOK undulator was patterned after the SSRL BL VI wiggler, but was intended for a laser harmonic experiment where error control and high-brightness harmonic output performance were the major issues. It was installed at the NSLS VUV storage ring in 1987. This neodymium-iron- 
boron/vanadium Permondur hybrid device also achieved random error performance of 0.5 percent or better. The most recently built device studied is the BL $\mathrm{X}$ wiggler. Installed in 1987, this is a high-field Nd-Fe/Permendur hybrid. Again, even though not intended for use as an undulator, it has a random errors of only 0.35 percent, making it an intereating candidate for this study. All these devices have mechanical drive systems allowing variable gap tuning of the insertion device field.

Extensive magnetic field measurements were made on these devices[7] [8] and formed the basis for the analysia done in this paper. The reader should refer to those references for the details of the measurement systems used. Fig. 3, Fig. 4, and Fig. 5 show on axis measurements of the vertical field of these devices plotting $B_{y}(z)$ versus $z$. $B_{y}$ is measured in gauss and $z$ is measured in $\mathrm{cm}$. These data were taken using precision Hall probe and translation stage syatems for particular gap settings for each device, $16.3 \mathrm{~mm}$ for BL VI, $30 \mathrm{~mm}$ for TOK, and $21 \mathrm{~mm}$ for BL X.

\subsection{Fourier analysis}

It can be seen in the figures that the field profiles are non-sinusoidal, with the triangular shape characteristic of the presence of $3^{\text {rd }}, 5^{\text {th }}$, etc. harmonics. The symmetry of the field map with respect to the $z$-axis indicates that even harmonics are weak, as required by the symmetry of the periodic magnetic structure. In fact, any strong even harmonic content in the data is an indication of a problem, possibly that the magnetic measurement system is not symmetrical in its response to positive and negative fields. The odd spatial harmoxic content is a natural consequence of the periodic magnet design. Small purely periodic field harmonics have only a minor effect on the radiated spectral output of the insertion device at a given spectral harmonic.

As a first step in separating systematic from random errors, the Fourier transform of the data in Figures 3-5 is performed. $A$ plot of the log of the magnitude of the Fourier spectrum of the BL $X$ data is shown in Fig. 6 . The essentials of the process of producing this figure are as follows: multiply the raw data of Fig. 5 by a window function to suppress side lobe artifacts in the transform[9] , interjolate possibly nonuniformly spaced data onto a uniform mesh, pad with zeros, Fourier transform using the FFT algorithm, normalize the magnitude of the complex Fourier transform to unit 
first harmonic height, and take the base ten logarithm. The windowing operation also tends to reduce the weight of the non-periodic fields in the end region.

From the figure, it can be seen that the third harmonic is about ten percent of the fundamental, and that harmonics at least up to the ninth are significant above the noise. For smaller magnet gaps (not shown), harmonics up to the thirteenth are significant. The magnitude of noise level in the data seems to be at about the $5 \times 10^{-4}$ level, or about $66 \mathrm{db}$ down from the fundamental. At this level there is no visible $2^{\text {nd }}$, $4^{\text {th }}, 6^{\text {th }}$, or $8^{\text {th }}$ harmonic, indicating a very low level of +1 - measurement asymmetry, as mentioned above.

There also seems to be s slow variation in the noise level as a function of spatial frequency, with low frequencies up to about the third harmonic being enhanced somewhat. The spatial frequency content of random field errors is constrained by the fact that real fields must satisfy Laplace's equation in three dimensions. This results in a filtering or smoothing of random errors produced in the magnetic structure and measured on axis. Therefore, it is likely that the low frequency noise background is the Fourier spectrum of the random field errors, and that the high frequency noise represents the errors in the magnetic field measurements. The exact connection between the Fourier spectrum of measured data and the possible spatial frequencies in field errors has not been fully worked out, but it might be used to separate noise in the measurement process from true field errors[10] .

\subsection{Curve fitting}

Once it has been determined via Fourier analysis which spatial harmonics contribute significantly to the data, a curve fitting procedure using non-linear least squares can remove these systematic periodic fields from the original data, leaving only the random field errors and the measurement noise. In addition, the fitting procedure can also fit parameters that should be known already, such as the relative phases of the harmonics, and even the value of the period of the magnet, $\lambda_{w}$, as a check of the overall measurement and analysis process.

The results of such an analysis are shown in Fig. 7 , where only the fitting residuals, $\epsilon(z)$, are plotted as a function of $z$. The residuals look quite random, with 
no periodic components visible. As a check of this, the Fourier transform of the residuals can be examined. In this case, only broad bend noise, with some emphasis on low frequencies as before, is observed. The large quasi-periodic residual between about $25 \mathrm{~cm}$ and $50 \mathrm{~cm}$ will be examined in more detail in a moment. Also observed is a rising fit residual at each end of the plot. This is the exponentially decaying end effect caused by the termination of the periodic magnet structure. In the fit, the non-periodic ends have been chopped off.

For pure periodic signals it can be shown that least squares fitting of sinusoids is equivalent to Fourier analysis. Therefore, a Fourier filtering approach to the random error extraction process shoulf also be possible.

An expanded view of the region around the large quasi-periodic residual seen in Fig. 7 is shown in Fig. 8 . Here, the pole positions have been indicated by dotted lines. It is interesting that the peaks in the random field error seem to be between the pole positions. A similar effect is seen in Fig. 9 for the TOK undulator data. In this case, almost all of the peak values of the residual random errors lie between the pole positions.

During the manufacture of this magnet, the lack of understanding of the source of observed field errors resulted in a great deal of effort to ensure that the poles were in the proper positions, with shimming performed to bring the pole tip surfaces for each half of the undulator into the proper planes to within a tight tolerance of $\pm 2.5 \mu$. Only later were the data analysis tools developed that showed the errors to be mainly between the poles.

Once this effect was discovered, and misoriented magnet blocks identified as the probable culprit, pole assemblies were rearranged to place large positive field errors next to large negative ones, thus introducing correlations into the random errors and appraximately cancelling out large beam deflections. As is discussed in Ref. [2], such correlations reduce the effect of errors on the radiation spectrum. For the TOK undulator, this reduction was approximately a factor of five, resulting in an effective error of 0.1 percent RMS, even though the true RMS error was 0.5 percent. This extra rearrangement of pole assemblies resulted in the unusual correlated or quasiperiodic appearance of the fit residuals seen in Fig 9. The Fourier transform of the 
reaiduals in Fig. 9, however shows only a broad band spectrum, with no sharp periodic components, consistent with a truly random error.

\subsection{Integrated field errors}

The existence of integrated dipole, quadrupole, sextupole, and higher order multipole fields in hybrid undulators is unusual, since, according to the symmetry of the magnetic structure, a properly manufactured hybrid device should possess no auch integrated multipolea, and any such fields obwerved experimentally must be due to $n$ field error of some kind.

By integrated multipoles it is meant that as a function of transverse position, $x$ (horizontal) (or $y$ (vertical)), the field integral $I(x)=\int B(z) d z$ has a constant (dipole), linear (quadrupole), or quadratic (sextupole) dependence on $x$. A periodic hybrid undulator contains no real dipole, quadrupole, or sextupole fields but integrated fields can satisfy a 2-D Laplace equation, 20 an integrated multipole model makes sense.

Certain kinds of design errors or manufacturing tolerances are known to produce integrated multipole errors. For example, if the steel backing beams of the top and bottom hali of the undulator structure are not connected together magnetically an integrated dipole field is possible. Similarly, if the steel poles have tilt misalignment errors with respect to the midplane of the magnet, an integrated quadrupole field may be produced. If the ends of the periodic magnetic structure are not properly terminated, a quadratic, or sextupole, varjation of field integral with transverse position is possible. In addition, imperfections in the active magnetic material can produce any of the multipoles described, both in a normal or rotated (skew) configuration.

Integrated dipole fields can deflect the electron beam in the insertion device. This kick results in a closed orbit displacement in the stored electron beam. In addition, within the undulator itself, the normally straight electron trajectory becomes curved, something along the lines of the average curvature obeerved in Fig. 1. This curvature reduces the brightness of the radiated beam.

Integrated quadrupole errors can change the horizontal or vertical tune of the storage ring or can introduce $x-y$ coupling. In addition, if the electron beam is placed 
somewhat off center in the insertion device, the integrated quadrupole error looks like a dipale and steers the beam. This results in a closed orbit distortion that can be larger than is acceptable[11] .

In Fig. 10 is shown the variation of the field integral for BL $\mathrm{X}$ as a function of $x$. Note that the variation is not a simple constant, linear, or quadratic function. Also note that if one only looks near $z=0$ that the linlar (quadrupole) term is quite large. It is clear that this behavior cannot be explained using the simple mechanical tolerance arguments given above.

\section{Location of field errors}

We have seen in previous sections indications that random field errors are localized between the steel poles.

\subsection{Difference maps}

In an attempt to discover the origin of the unusual variation in field integral shown in Fig. 10, difference maps were calculated. In Fig. 11 is plotted the difference between two field measurements, $\Delta B_{y}(z) \equiv B_{y}(x=+16 m m)-B_{y}(x=-16 m m)$, as a function of $z$. This analysis method does not depend on any particular model of the data in terms of spatial harmonics, but only depends on the stability and repeatability of the measurement system. The large peaks between steel pole positions are probably due to differences in non-easy-axis magnetization in the Nd-Fe blocks nearest the electron beam. If 80 , this is an indication that controlling this parameter of the magnetic material is important.

The field difference map has relatively small values directly under the poles, consistent with the shielding properties of the steel poles against transverse fields. This is one of the major advantages of the hybrid undulator design over the previous allpermanent-magnet design, where there is no steel structure enforcing uniformity of field boundary conditions near the electron beam.

If one integrates the difference map in Fig. 11, Fig. 12 results. This shows that the field integral difference $\int \Delta B_{y}(z)$ is not localized in a particular region of 
the magnetic structure, but instead builds up more or less uniformly as a function of $z$. This shows that the integrated field error variation with transverse position is not caused by one single bad block or peak in the difference map, but is distributed in a more or less randonily uniform way. This means that an attempt to correct the latesal variation of field integrals using some local correction method such as tuning studs or local mechanical shims is inappropriate.

\subsection{Integrated errors}

In the previous section it was suggested that somehow the variation of field integral with transverse position was coming from fields produced by misoriented or non-uniformly magnetized blocks near the midplane. A method of quantifying this statement is to try to separate this variation into two pieces, one coming from under the poles ("in-phase") and the other from between the poles ("quadrature-phase").

If one multiplies the raw data shown in Figs 3-5 by $\sin ^{2}\left(2 \pi z / \lambda_{4}\right)$ and $\cos ^{2}\left(2 \pi z / \lambda_{4}\right)$, the two resulting data arrays represent, respectively, just the separation desired. In addition, the sum of the two arrays is just equal to the original data, so no information is lost in this sepercation. If one then computes the field integrals for each component, Fig. 13 results. This figure is fairly conclusive proof that fields located between the poles are the culprits in the unusual transverse variation of field integral for BL X.

Fig. 14 and Fig. 15 show the corresponding plots for BL VI and TOK, respectively. For BL VI, the ratio of the two variations is not so great, but is still pronounced. For the TOK, the variation of field integral is only slightly greater for the quadrature-phase component, and, as seen in Fig. 9, the TOK has the largest RMS field error, with the error fields localized primarily between the poles.

\section{Suminary}

The high performance expectations of the next generation of undulator-based synchrotron radiation sources place stringent performance and error tolerance specifications on the insertion devices. In an attempt to identify sources of errors in permanent magnet insertion devices, a study has been made of three existing highperformance hybrid devices. The devices were designed and built during a period of 
over five years, but some design principles were common to all of them. The analysis of magnetic measurement data shows several things. First, there is an absolute necessity for the highest quality of magnetic measurement data to meet the tight specification tolerances of the new undulators. Second, existing devices seem to have most of their field errors, both random and systematic, located between the steel poles rather than either under the poles or uniformly distributed along the magnet.

Some of the reaults of this analysis can be represented on a universal graph derived from the nemi-analytic theory of random errors in undulatrrs given in Ref. [2], as shown in Fig. 16 . The solid lines $F_{\Delta}=0.7$ and $G_{\Delta}=0.7$ represent 70 percent performance boundaries. For points to the right of either line, more than 30 percent undulator radiation spectral performance loss is incurred. The vertical and horizontal axes represent combinations of the parameters of the undulator, with $\sigma$ being the RMS fractional field error, $\boldsymbol{N}$ the number of periods, $n$ the undulator harmonic number, and $\boldsymbol{K}$ the deflection parameter. The solid dots on the graph represent the values of $p$ and $g$ for harmonics 1,3,5, etc. For more details, see Ref. [2]. This figure shows that all the devices analyzed in this paper could function well as undulators producing near ideal output at the first few harmonics. Some devices, although designed as wigglers, should work well as undulators, within the limitations imposed by the emittance and energy of the storage ring providing the electron beam.

\section{Conclusions}

In considering either the field errors (fit residuals) alone or their integrated effect, the imbalance between in-phase errors and quadrature-phase exrors means either that the errors between the poles have reaulted from an uncontrolled parameter in the design of hybrid magnets, or that the tolerances on pole positions and other arrors arising under the poles las been overly conservative, considering that most of the total error is coming from another source.

It is possible that the relative in-phase and quadrature-phase field integral variation is influenced by other differences among the devices studied, such as the gap-toperiod ratio, $g / \lambda_{w}$, the pole width-to-gap ratio, the pole thickness-to-period ratio, and the detailed angular and spatial distribution of misoriented blocks. In addition, some 
of the variation of field integral may be due to the design of the end poles of the magnets and have nothing at all to do with field errors between the poles. Since the field integral variations seen in these devices represent fairly large integrated quadrupole and sextupole effects, it is clear that more experimental and theoretical work on the effect of magnetic material errors on hybrid undulators is needed to quantify these effects.

The design methods for hybrid insertion devices have, in the past, placed more emphasis on the control of pole dimensions and positions and on the matching of total magnetic moment of magnet blocks on each pole than on the control of errors due to misaligned or imperfect blocks located near the midplane. This has resulted in the present high level of performan: $i$ in existing hybrid devices.

However, it is clear that rnore emphasis needs to be placed on the control of the dominant error in the devices, the quadrature errors. These errors have a direct steering effect on the electron trajectery, and hence cause radiation performance loss. The errors introduced by improper choice of total magnetic moment, however, have an antisymmetric character, producing no steering of the electron beam, and correspondingly little loss of output performance.

Recently, measurements on surface fields produced by non-easy axis misorisntation and magnetization non-uniformity in Nd-Fe blocks have been performed[12] . These measurements must become part of the block surting and selection process in new insertion device designs. This is the same problem, but on a different scale, as that facing the designer of pu.e permanent magnet insertion devices. In hybrid devices, the precision periodic arrny of steel poles provides some shielding of error fieldis and enforces certain field boundary conditions, making certain block errors invisible to the electron beam.

Another area for future work is the possibility of designing a magnet with error control as the primary design criterion, sacrificing peak field performance for smaller errors.

There is therefore an opportunity to dramatically improve the error performance of hybrid insertion devices by controlling the quadrature-phase error, while at the same time possibly relaxing somewhat the tolerances on in-phase errors. 


\section{T. Acknowlodgements}

The author acknowledges with plensure many helpful conversations with $K$. Halbach and W. V. Hansenranil. 


\section{REFERENCES}

1) K. Halbach, "Fermanent Magnet Undulators" in Proceedings of the 1982 Bendor FEL Conference, Journal de Physique 44, p C1-211 (1983).

2) B. M. Kincaid, "Random Errors in undulators and their effects on the radjation spectrum" J. Opt. Soc. Am. B, 21294 (1985).

3) U5.0 Conceptual Design Report, LBL PUB-5256, Lawrence Berkeley Laboratory, University of California, Berkeley, CA 94720, Nov. 1989.

4) E. Hoyer, et al., "The Beam Line VI REC-Hybrid Wiggler for SSRL", IEEE Trans. Nucl. Sci., NS-30 3118 (1983).

5) A. M. Fauchet, B. C. Craft, J. N. Galayda, H. Hsieh, A. Luccio, J. B. Murphy, C. Pellegrini, A. van Steenbergen, G. Vignola, L. H. Yu, R. R. Freemen, and B. M. Kincaid, "Beamline U13-TOK", Brookhaven National Laboratories Annual Reports, 1985, 1986.

6) E. Hoyer, et al., "The Beam Line X Nd-Fe-Steel Hybrid Wiggler for SSRL", IEEE Catalog No. 87, Chap. 2387-9, 1508 (1987).

7) D. H. Neiron and M. I. Green, "LBL/SSRL/EXXON Wiggler, Results of 1983 Magnetic Measurements", LBL Electronics Engineering Note MT-327 (LBID 755), May 23, 1983.

D. H. Nelson, et al., "Magnetic Measurements for Tuning and Operating a Hybrid Wiggler", $8^{\text {th }}$ International Conference on Magnet Technology, September, 1983, Journal de Physique C1, Suppl. 1, 957 (1984).

M. I. Green and D. H. Nelson, "The Lawrence Berkeley Laboratory General Purpose Magnetic Measurement Data Acquisition System", loc. cit., 943 (1984).

D. H. Nelson, et al., "Magnetic Measurements of the LLNL/LBL/SSRL Beam-Line X Wiggler", LBL Electronics Engineering Note MT-378 (LBID 1300), Dec 16, 1987.

8) B. M. Kincaid, unpublished. 
9) R. B. Blackman and J. W. Tukey, "The Measurement of Power Spectra", Dover Publications, Inc., New York, 1958.

10) K. Halbach, private communication.

11) A. Jackson, private communication.

12) W. V. Hassenzahl, to be published. 


\section{FIGURE CAPTIONS}

1. Numerical simulation of random field error effects on the electron trajectory in an undulator with $K=2, N=50$, and $\sigma=0.005$. Here three different random number seeds have been used. The vertical axis is electron displacement normalized by the natural undulator oscillation amplitude, $a=\lambda_{4} K / 2 \pi \gamma$. The horizontal axis is $z / \lambda_{w}$. Presented in this way, the result is independent of $\lambda_{w}$ and $\gamma$ in the large $\gamma$ limit. The solid curves are the $1-\sigma$ envelopes of - large number of random trajectories. (See Ref. [2] for details).

2. Calculated on axis irradiance spectrum (power per unit solid angle and frequency) for the central electron trajectory shown in Fig 1. Here, $\omega_{1}$ is the frequency of the first harmonic in the undulator spectrum. The loss of relative intensity at the $5^{\text {th }}$ harmonic and the appearance of normally forbidden even harmonics on axis is caused by random field errors. (See Ref. [2] for details).

3. $B_{y}(z)$ vs. $z$ measured on axis for the BL VI wiggler.

4. $B_{y}(z)$ vs. $z$ measured on axis for the for the TOK undulator.

5. $B_{y}(z)$ vs. $z$ measured on axis for the for the $\mathrm{BL} \mathrm{X}$ wiggler.

6. The log of the normalized Fourier transform magnitude of windowed BL $\mathrm{X}$ date from Fig. 5.

7. Fit residuals for least squares fit of the central section of the BL $X$ field data. The residual errors look quite random, and have an RMS value of 0.35 percent of the main field.

8. An expanded plot of the BL $\mathrm{X}$ fit residuals for the quasi-periodic region shown in Fig. 7. The dotted vertical lines represent steel pole positions. Note that the fit residuals are large in the regions between the poles.

9. Fit residuals for the TOK undulator. Note that most of the residual peaks occur between the poles of the magnet (denoted by dotted lines). 
10. Plot of field integrals for $B L X$ as a function of transverse position. Note that the variation does not follow a simple quadratic polynomial. This means that an integrated multipole description of the field is of limited value.

11. Plot of the difference $\Delta B_{y}(z)$ for $B L X$ (see text). The dotted lines are the steel pole positiuns. Note the large number of peaks between pole positions.

12. Integral of field difference map shown in Fig 11. Note that the field integral difference is not localized at any one place in the magnet, but builds up more or less uniformly along the length of the device.

13. Plot of the total, the in-phase, and the quadrature-phase components of $\mathrm{BL}$ $X$ field integral. An arbitrary constant background has been removed from the in-phase and quadrature-phase components so as to fit all three curves on a single plot. Note that most of the variation in field integral comes from between the poles.

14. Plot of the total, the in-phase, and the quadrature-phase components of the BL VI field integral. Again, an arbitrary constant background has been removed from the in-phase and quadrature-phase components.

15. Plot of the total, the in-phase, and the quadrature-phase components of the TOK field integral. Again, an arbitrary constant background has been removed from the in-phase and quadrature-phase components.

16. Universal performance graph showing error performance of the three devices analyzed in this pnper and the proposed ALS US.0 undulator. derived from Ref. [2]. The two sets of points for the TOK correspond to the ectual RMS error of 0.5 percent and the effective error of 0.1 percent, accounting for deliberately introduced error correlations. See text for details. 


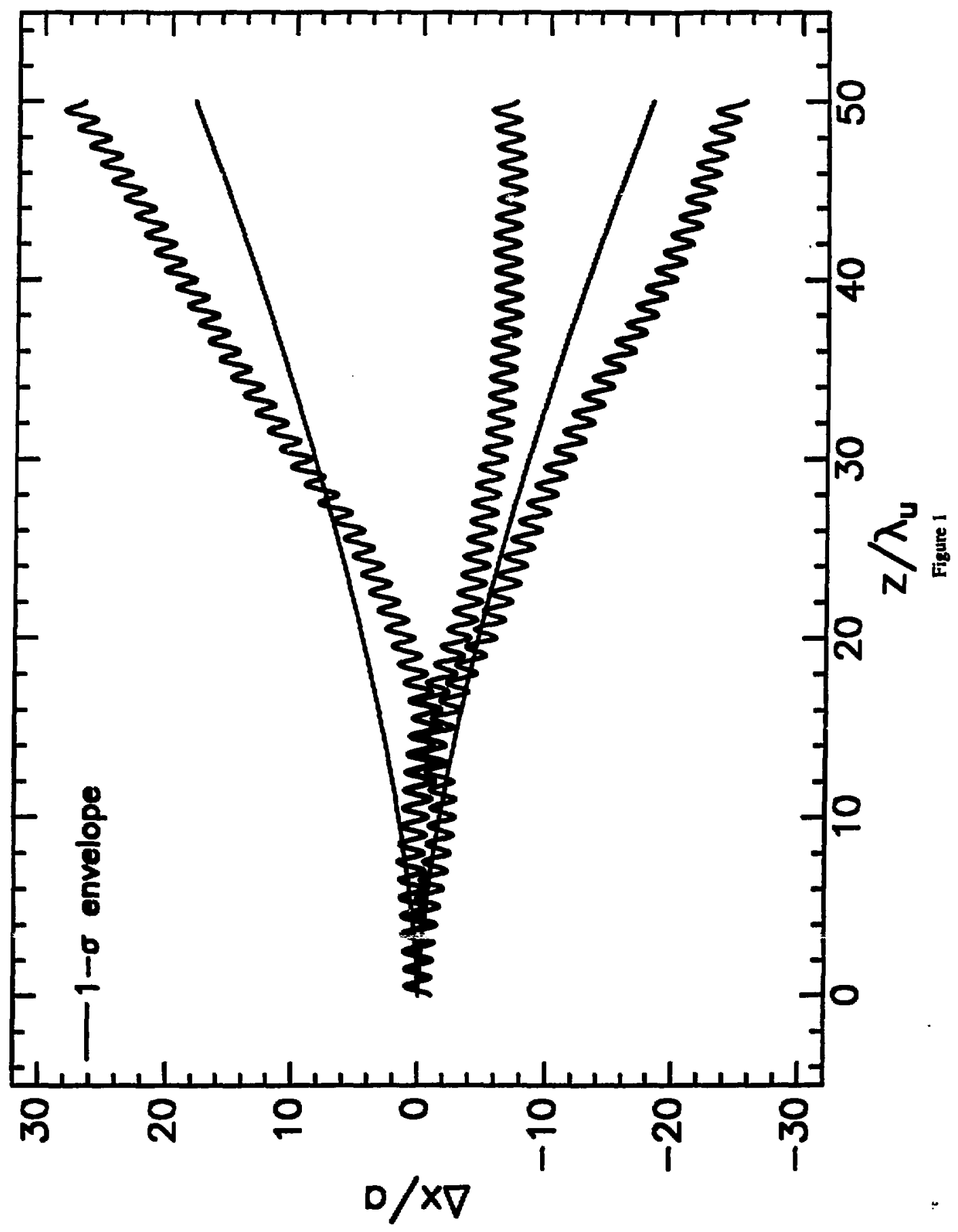




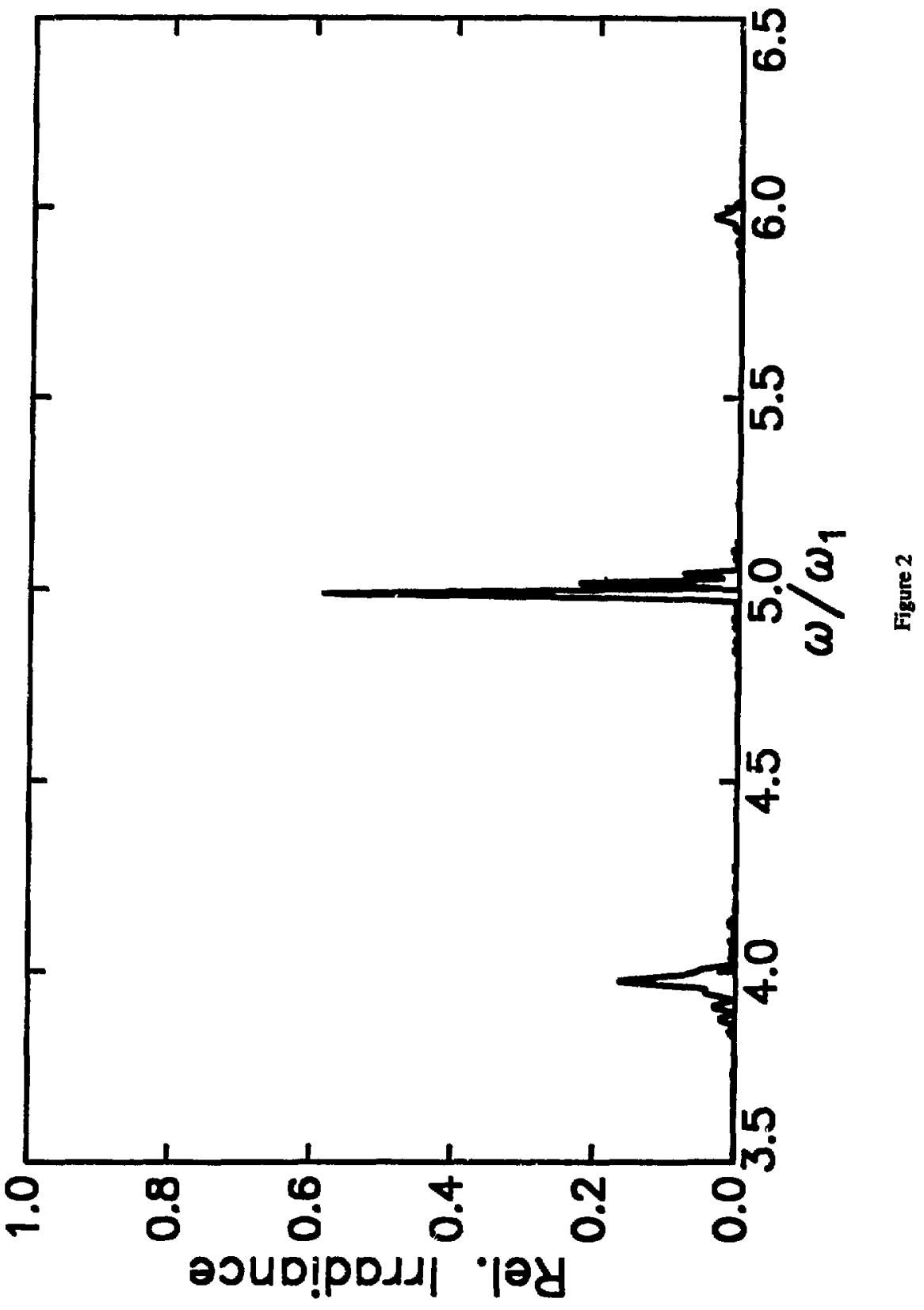




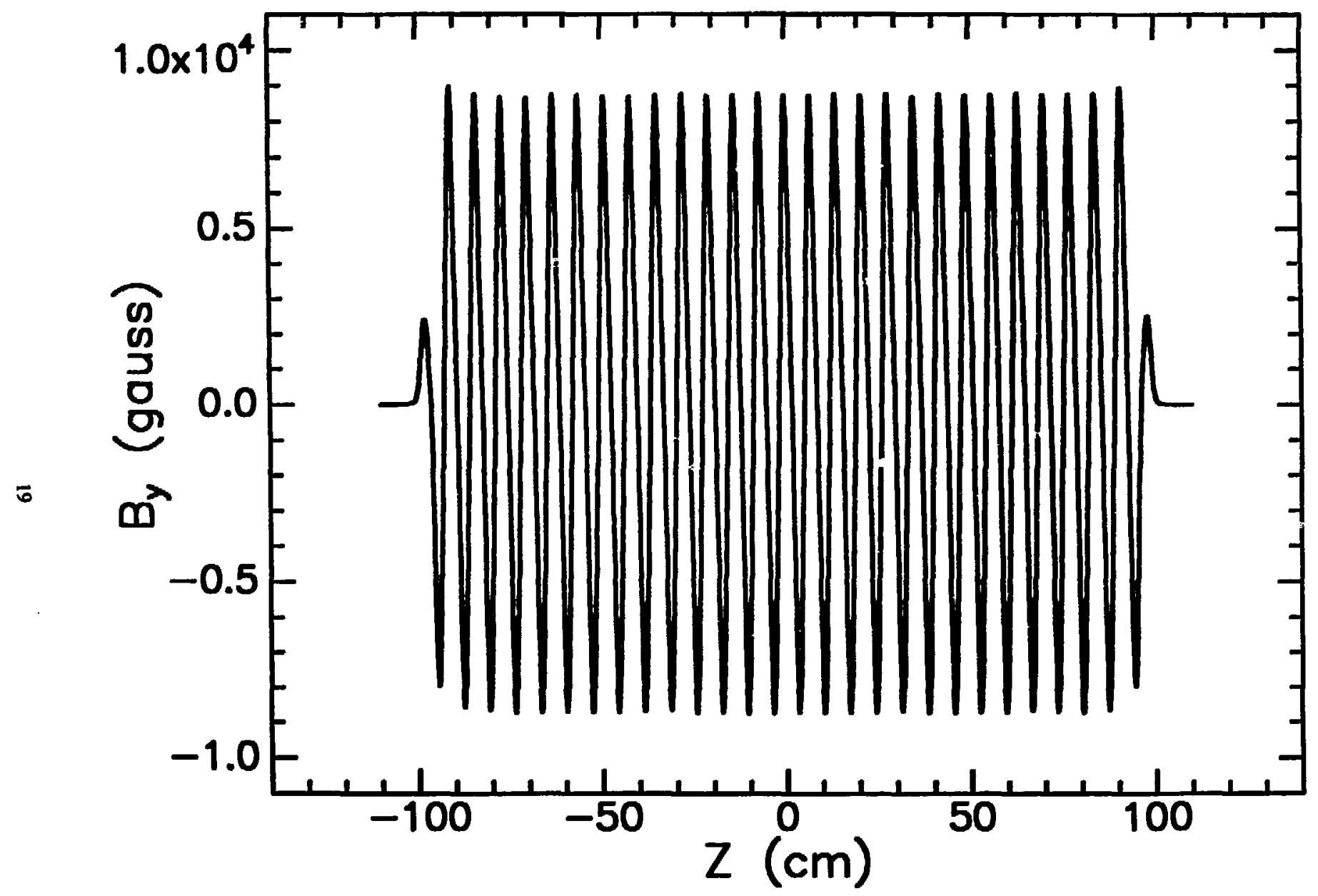

Figure 3 


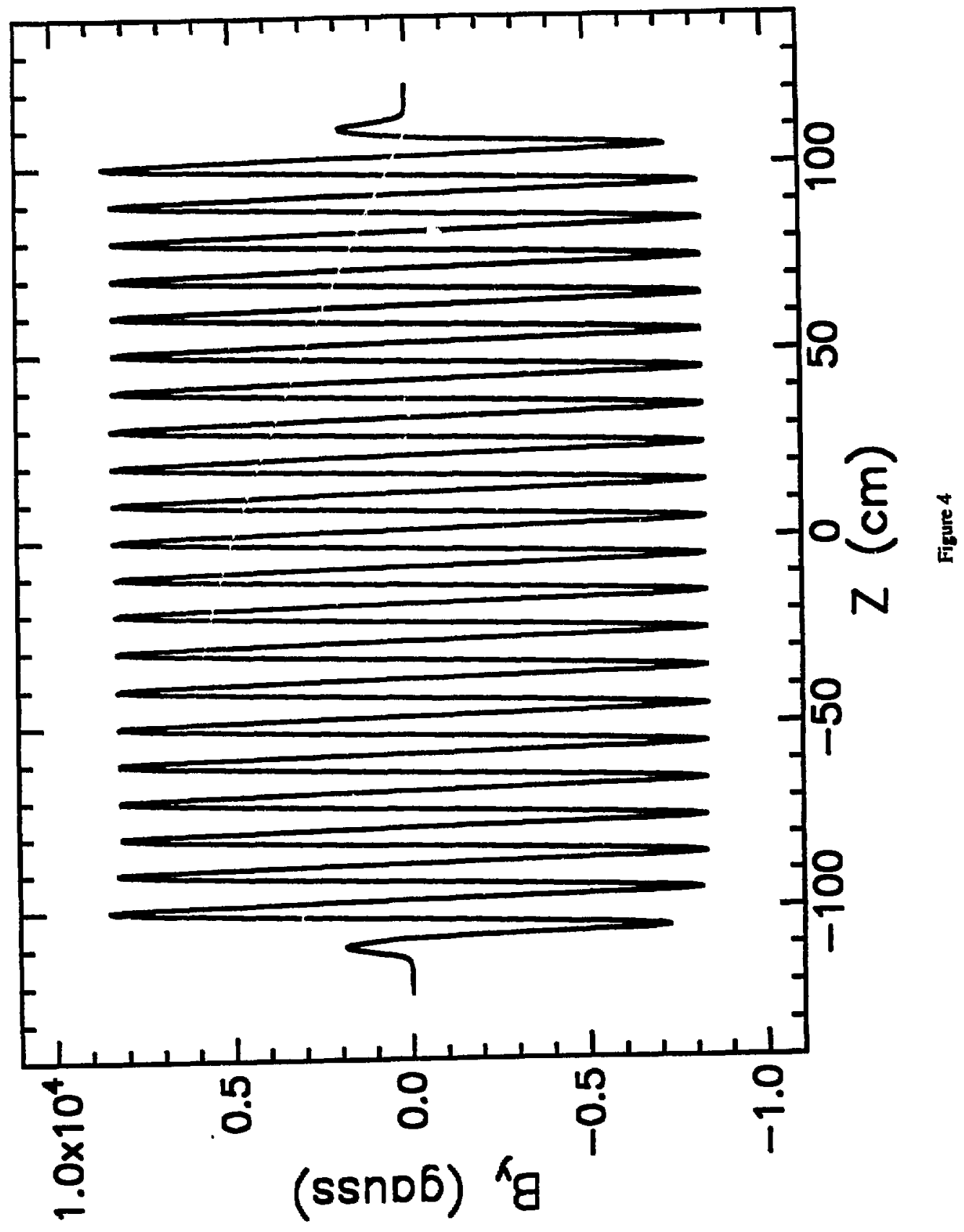




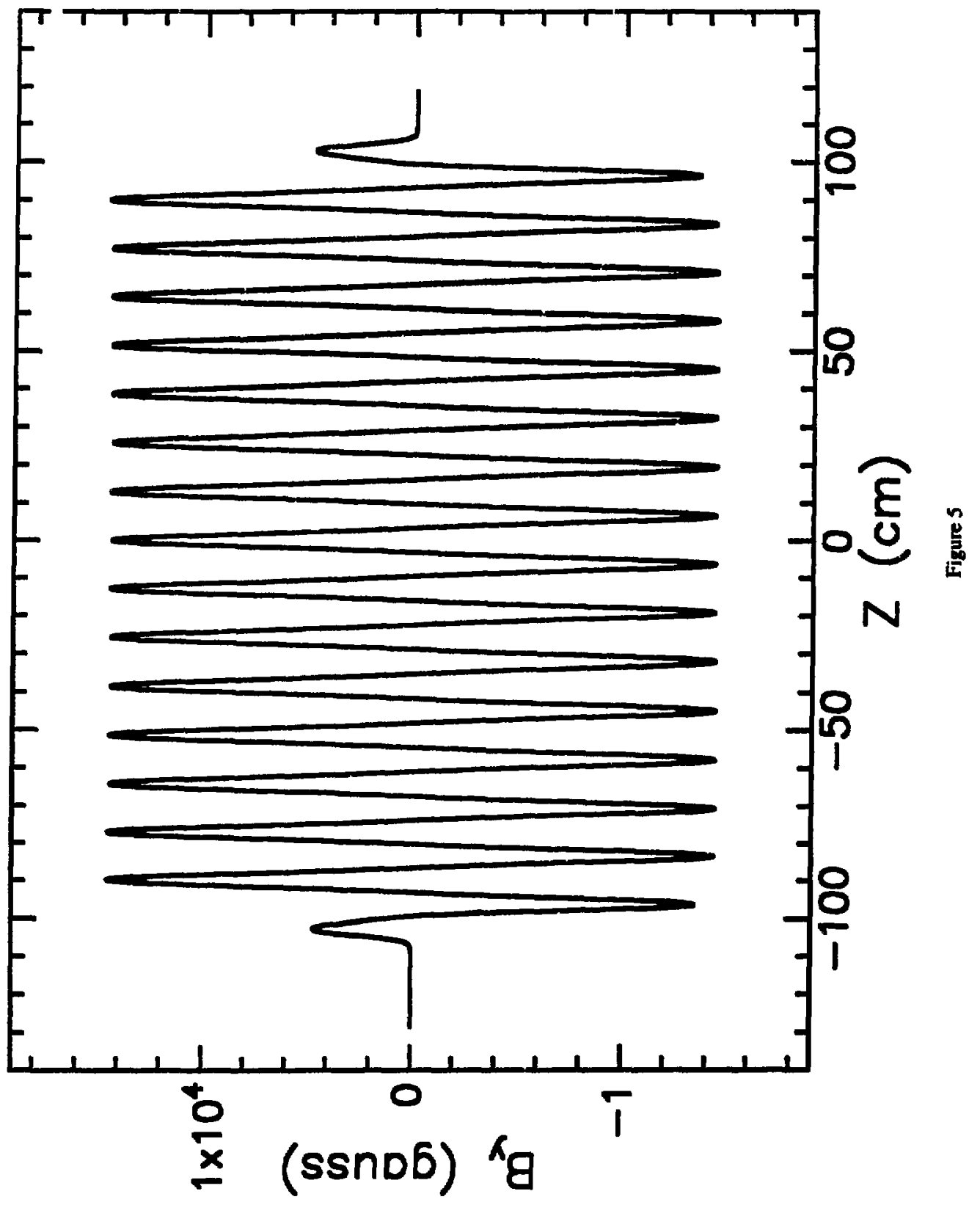




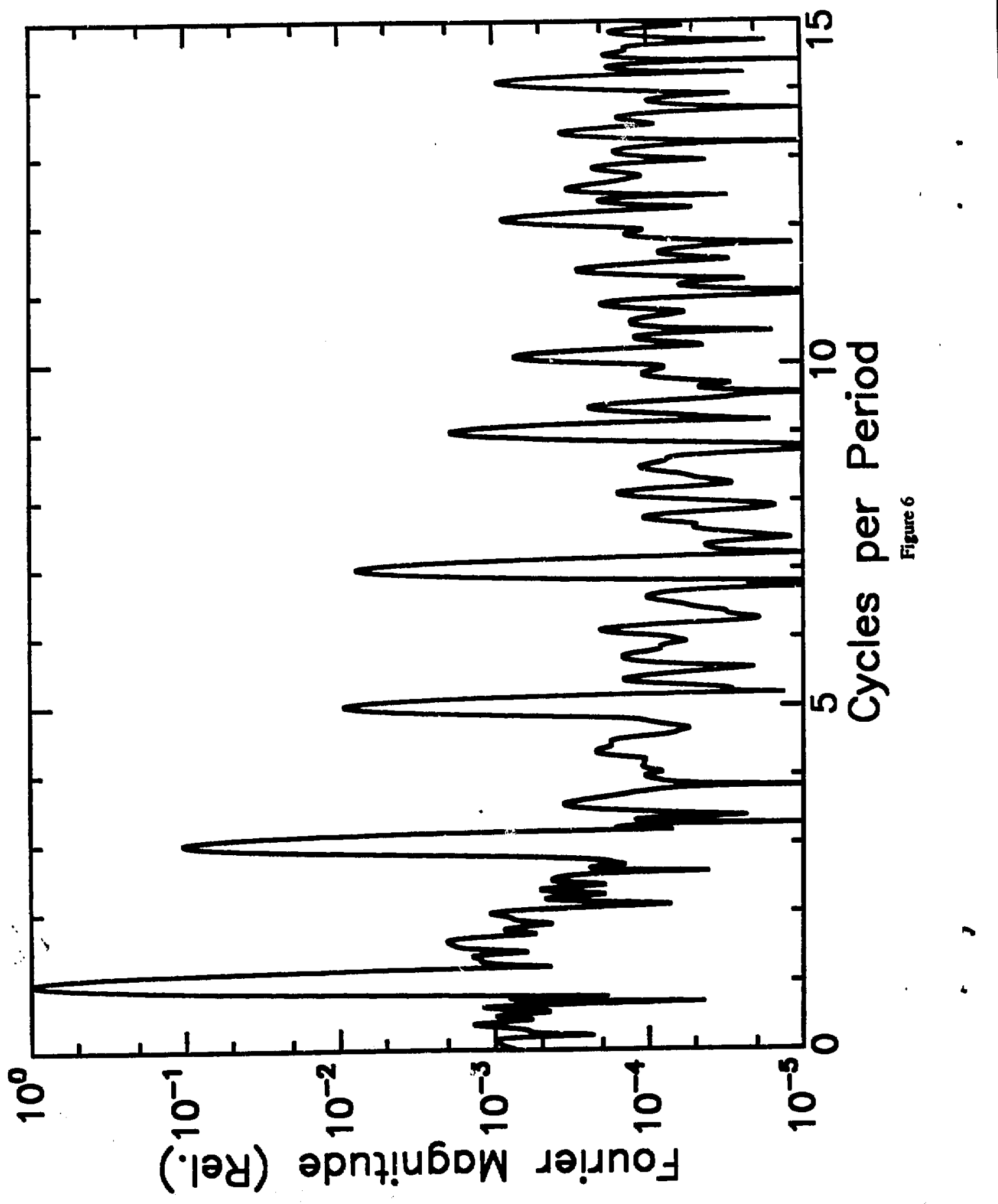




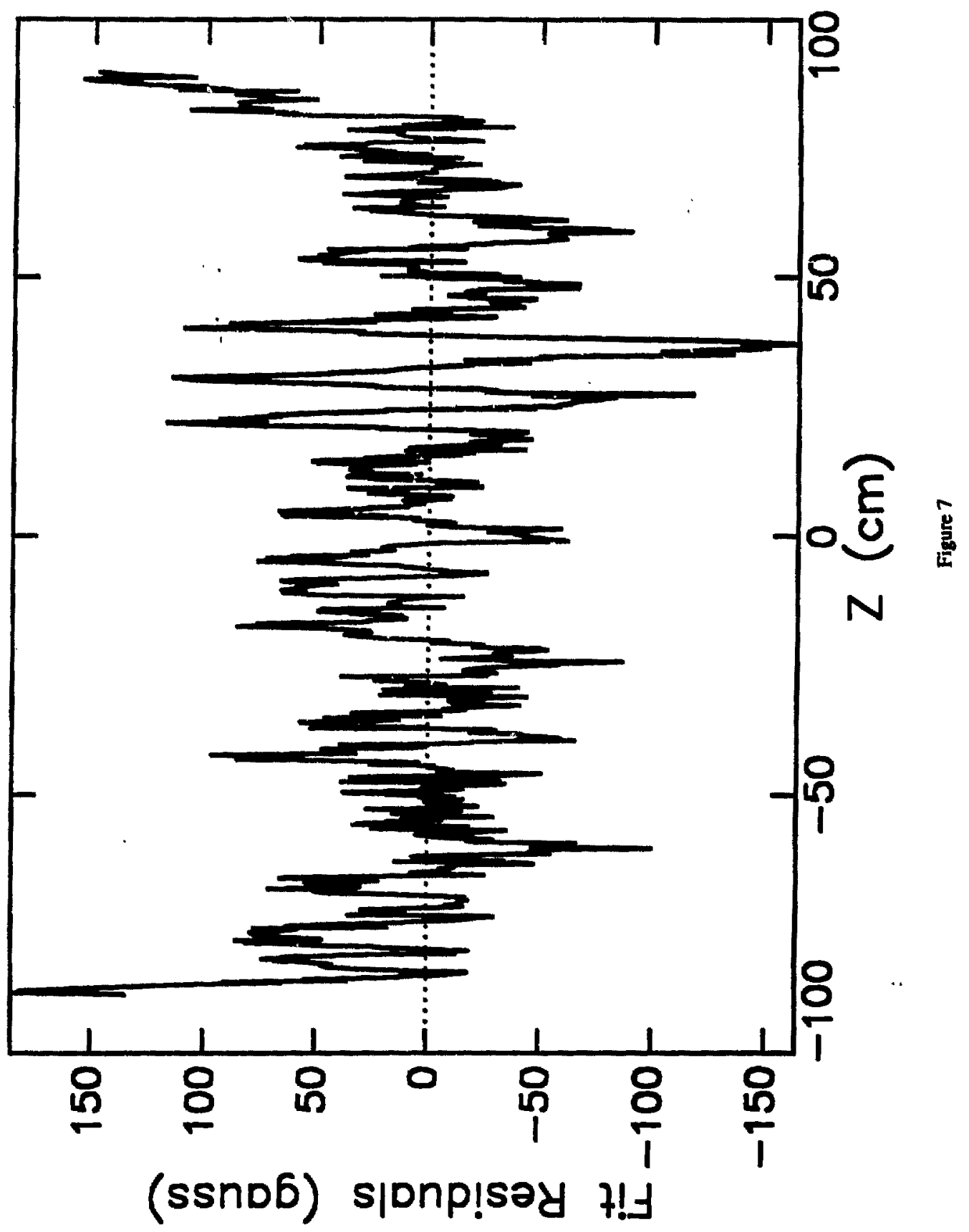




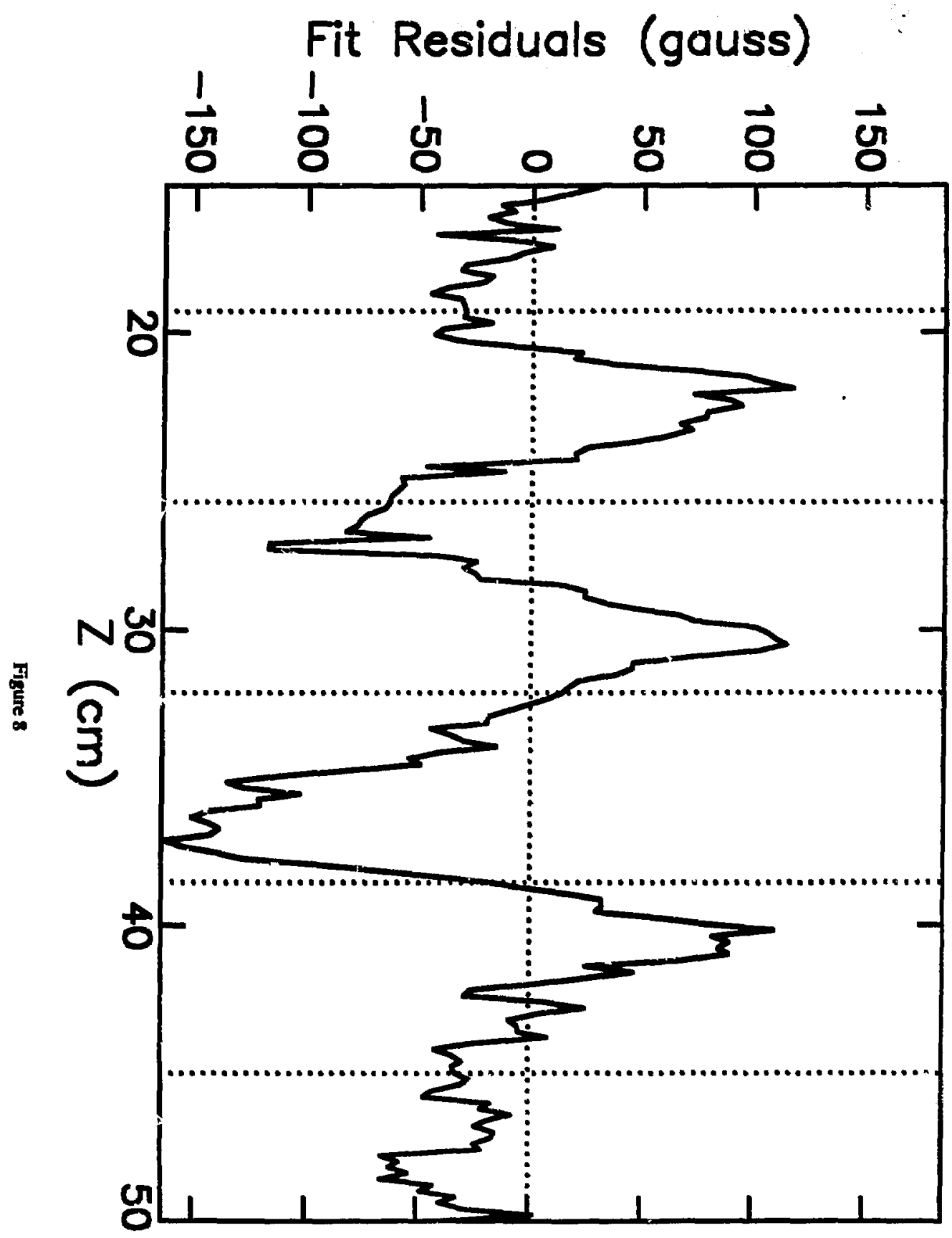




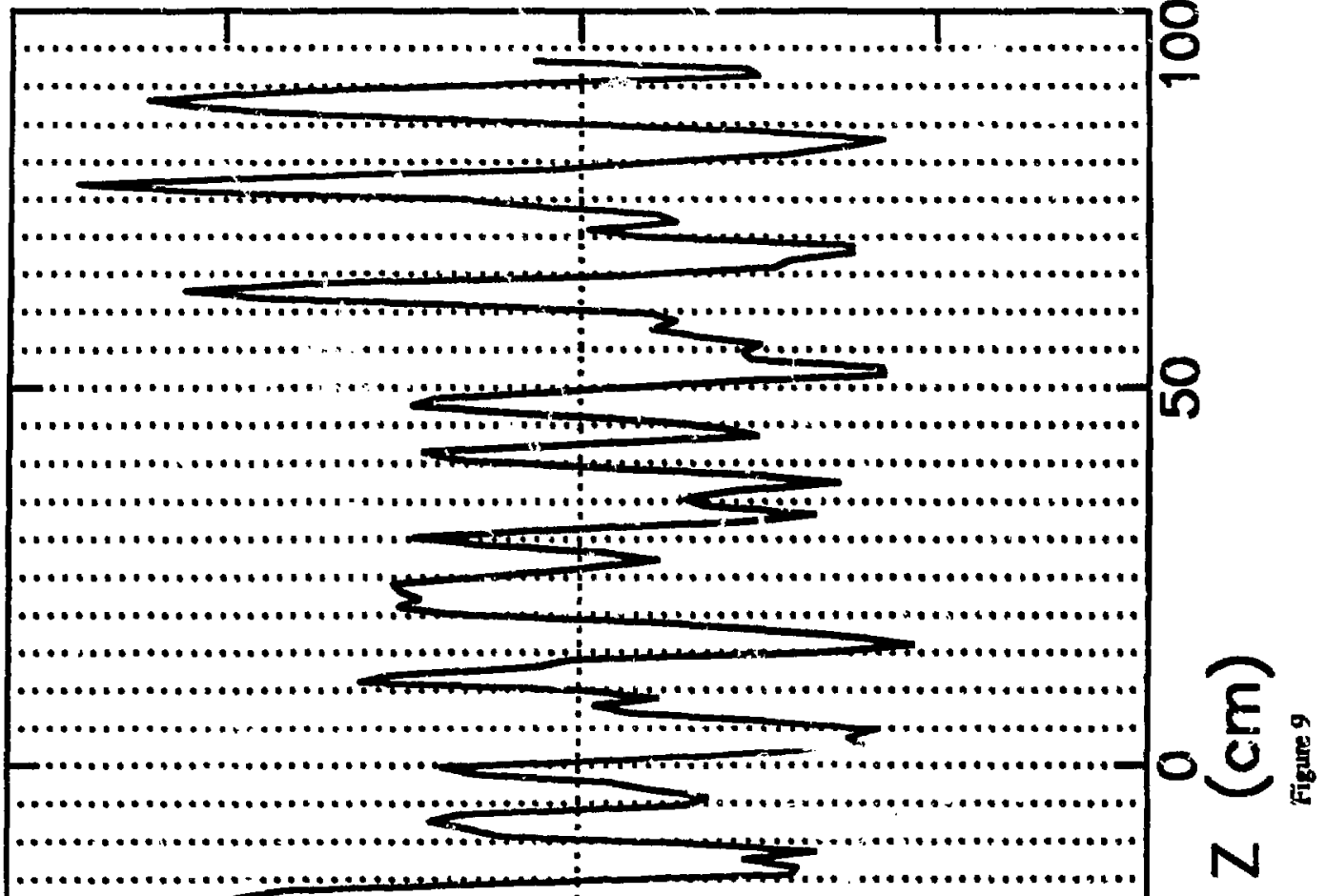

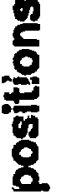

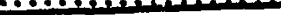

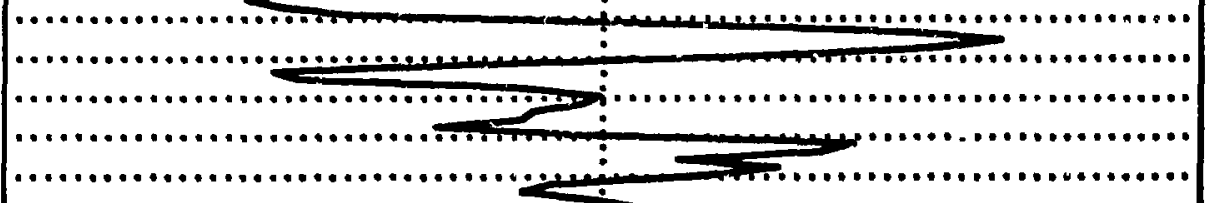

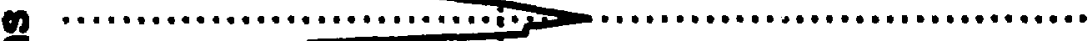

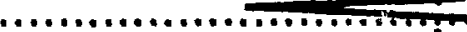
(1)

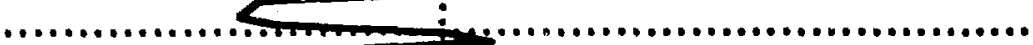

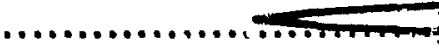

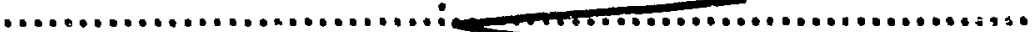
๑ $\ldots \ldots \ldots \ldots \ldots \ldots \ldots \ldots \ldots \ldots \ldots \ldots \ldots . . \ldots \geq \ldots \ldots \ldots \ldots \ldots \ldots \ldots \ldots$

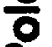
..............................

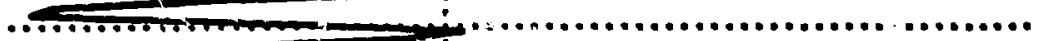

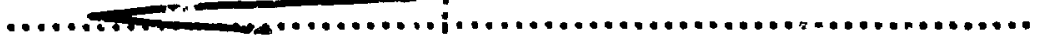

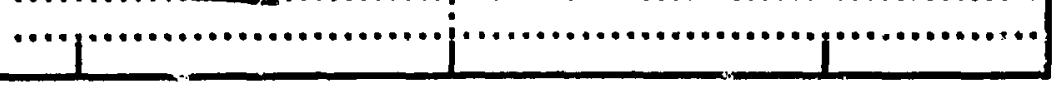
0 (1) (ssnd6) s|pnp!səy $7 !-4$ 


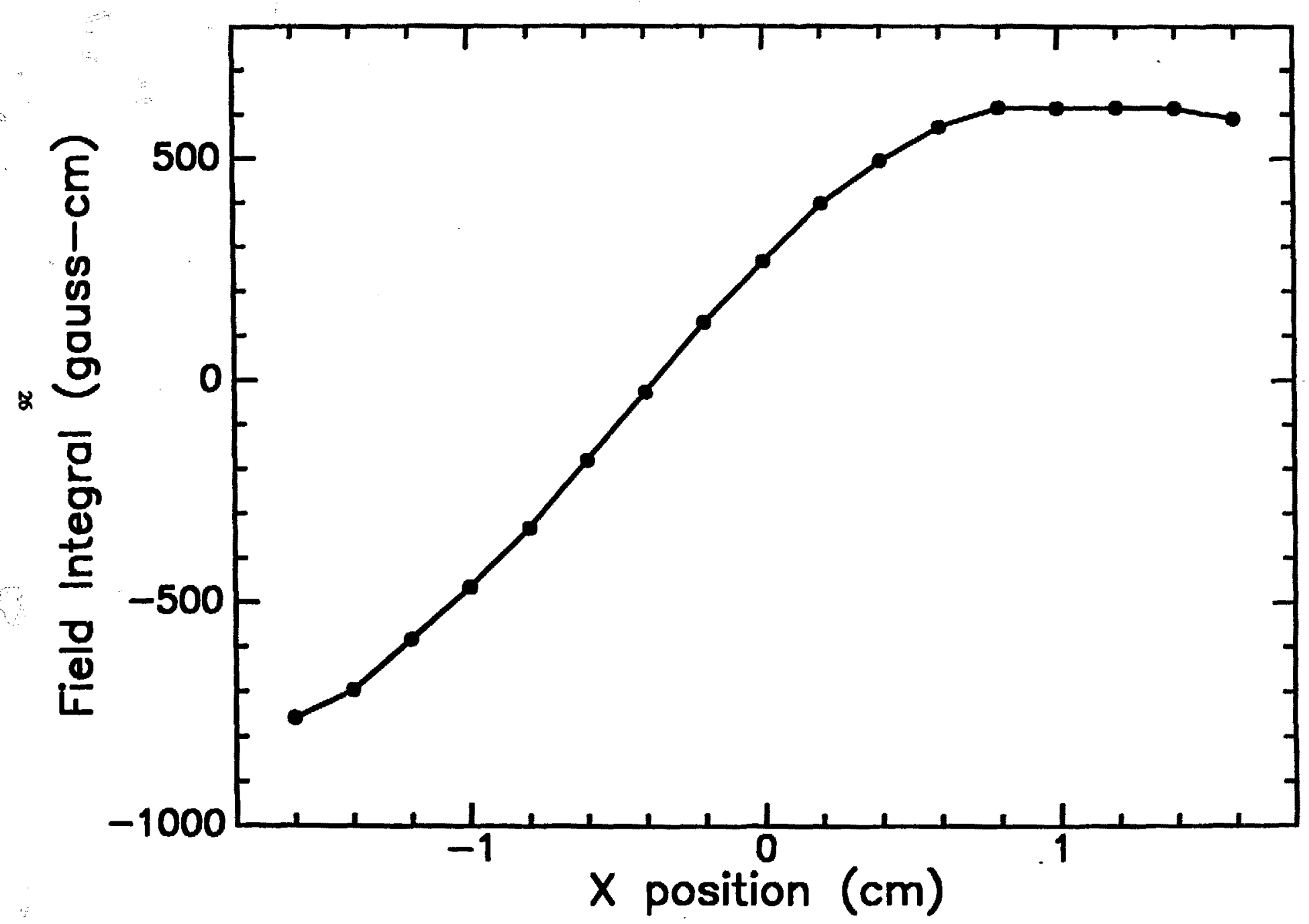

Figure 10 


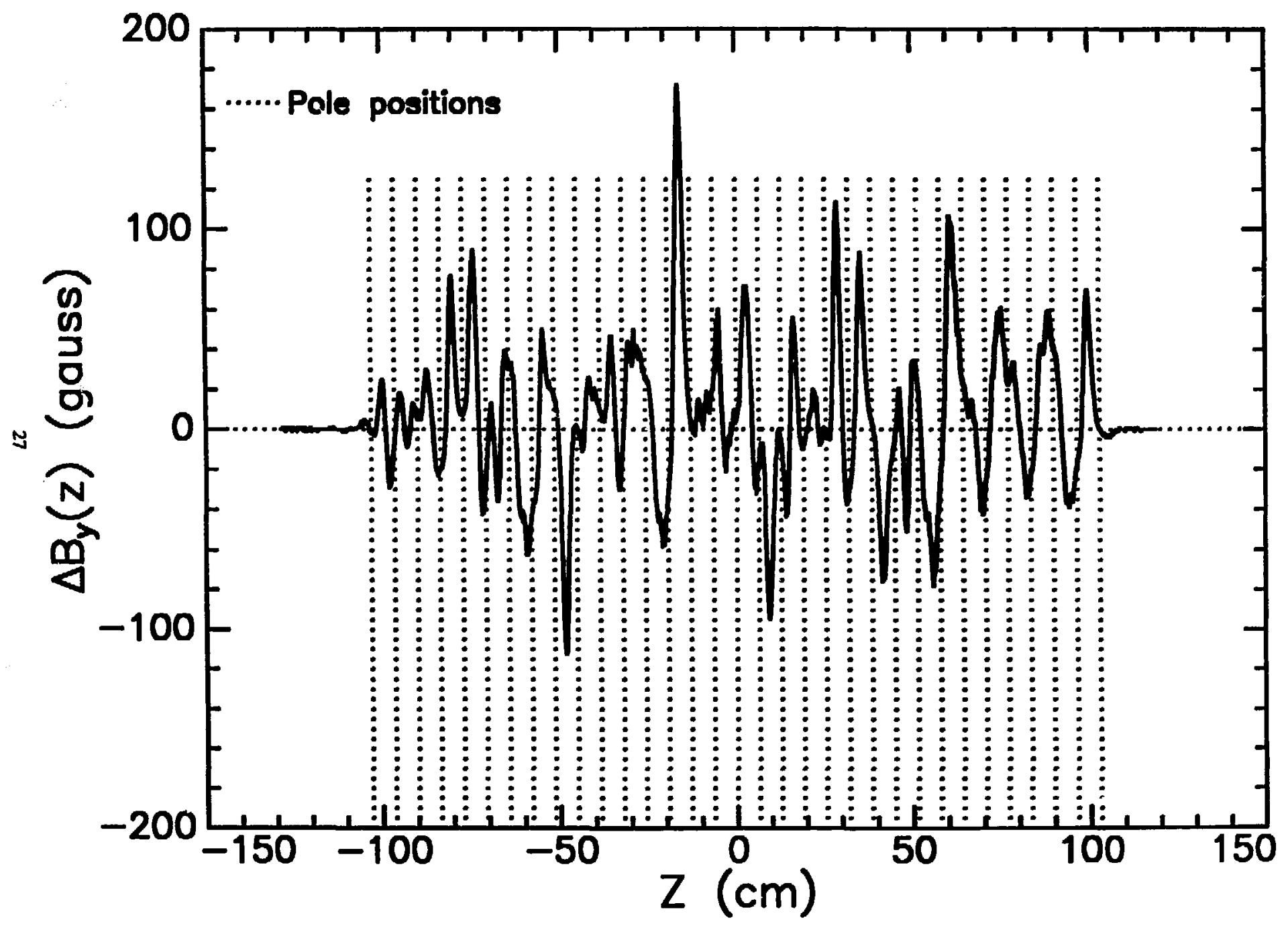

Figure 11 


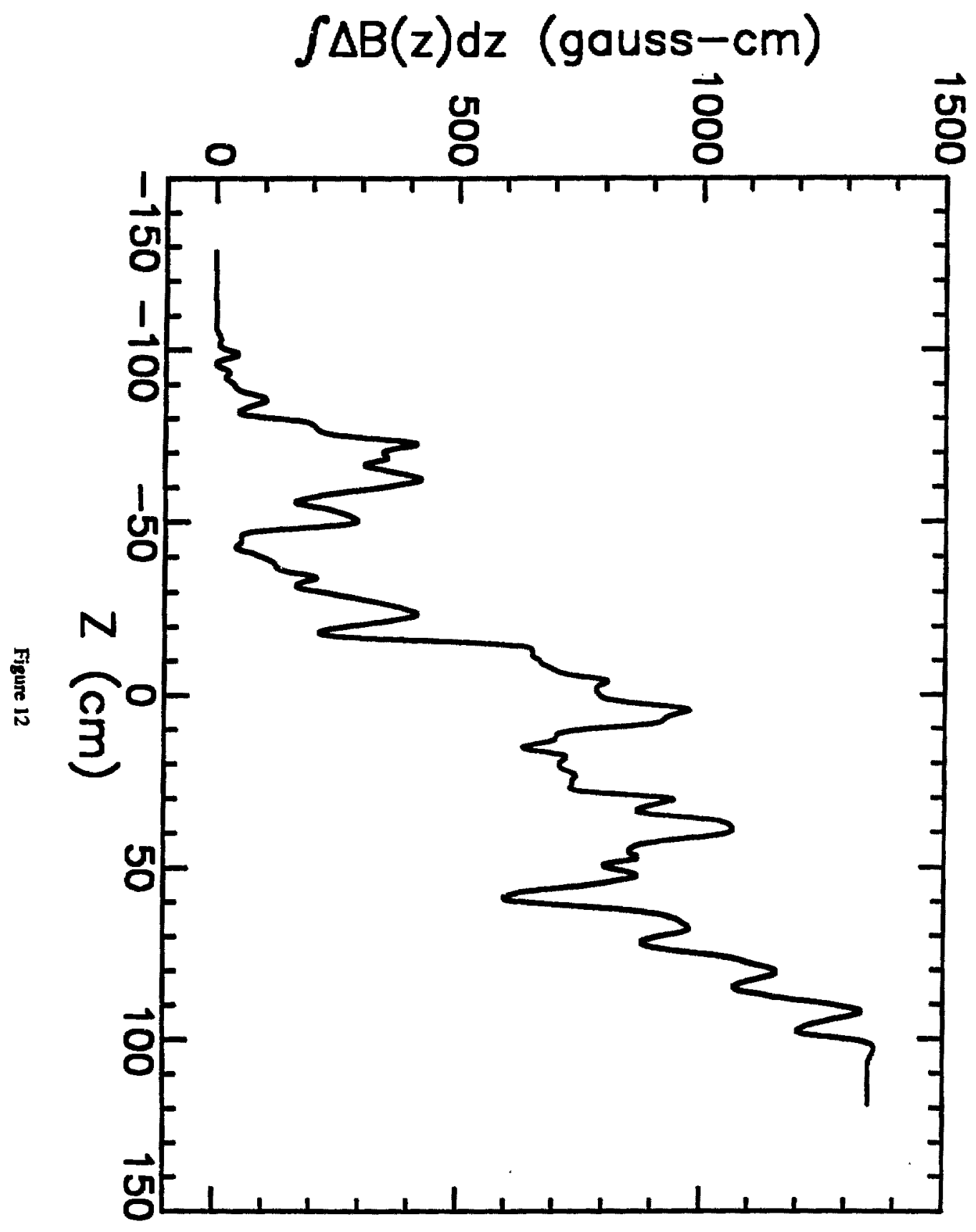




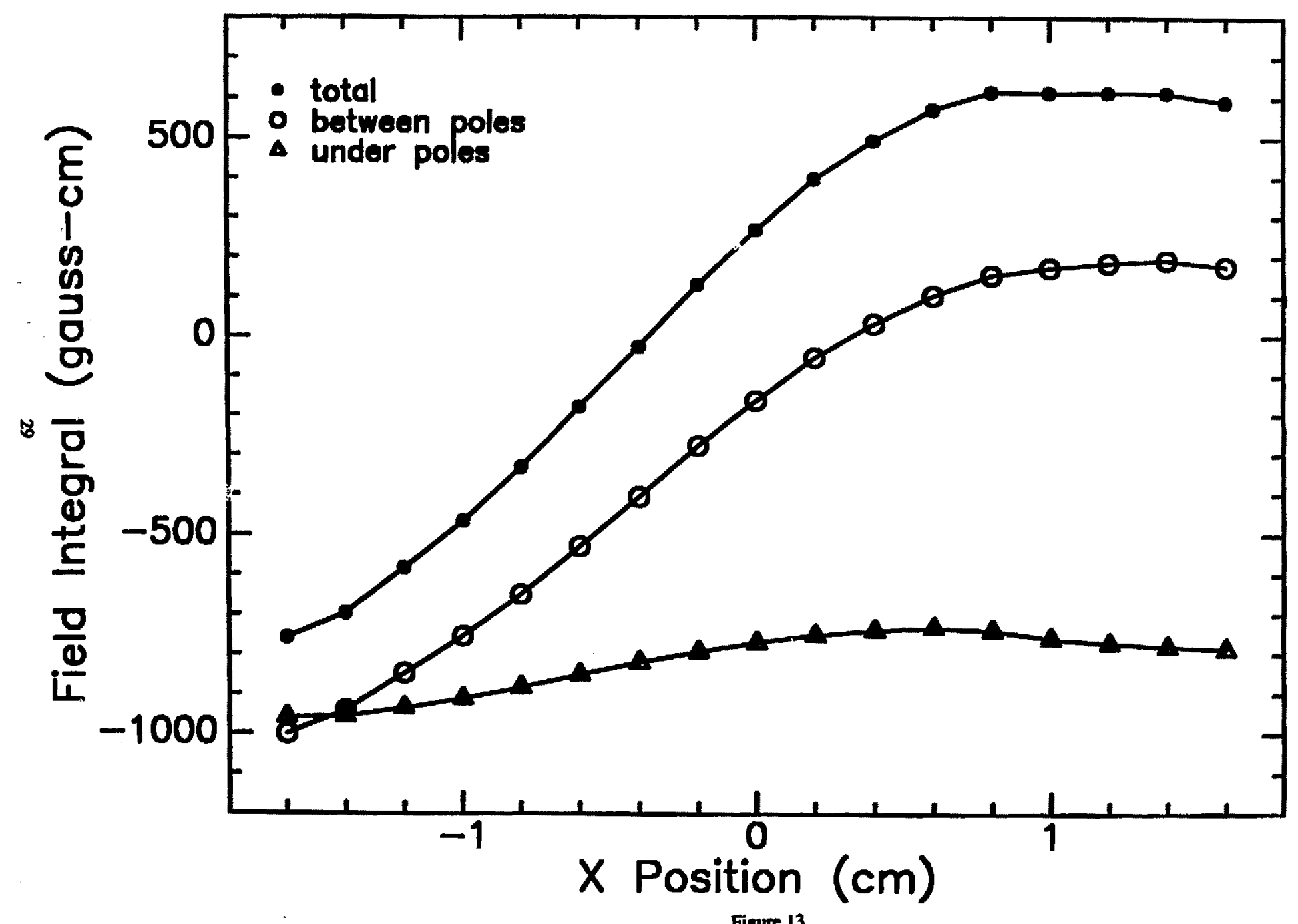

Figure 13 


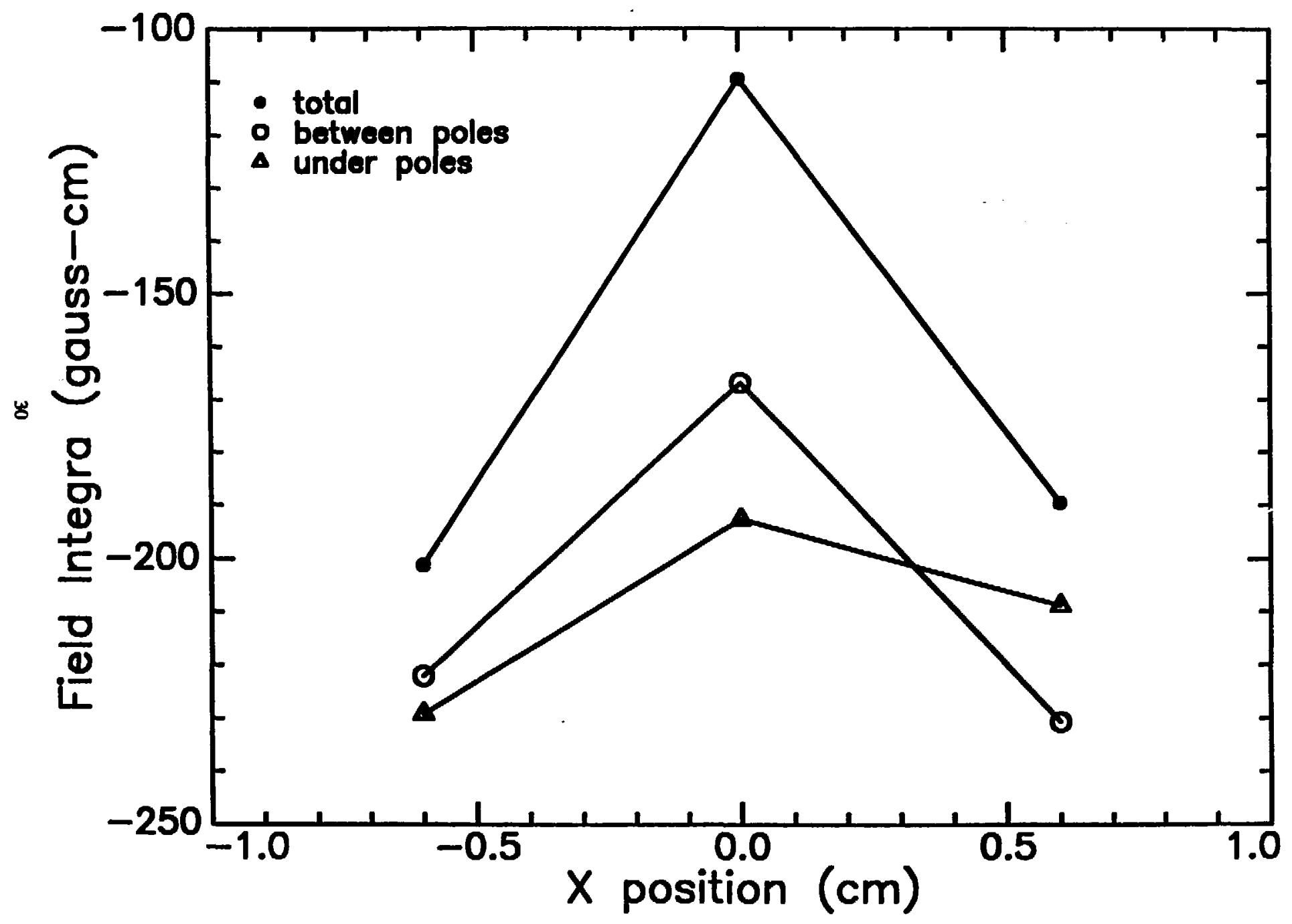

Figure 14 


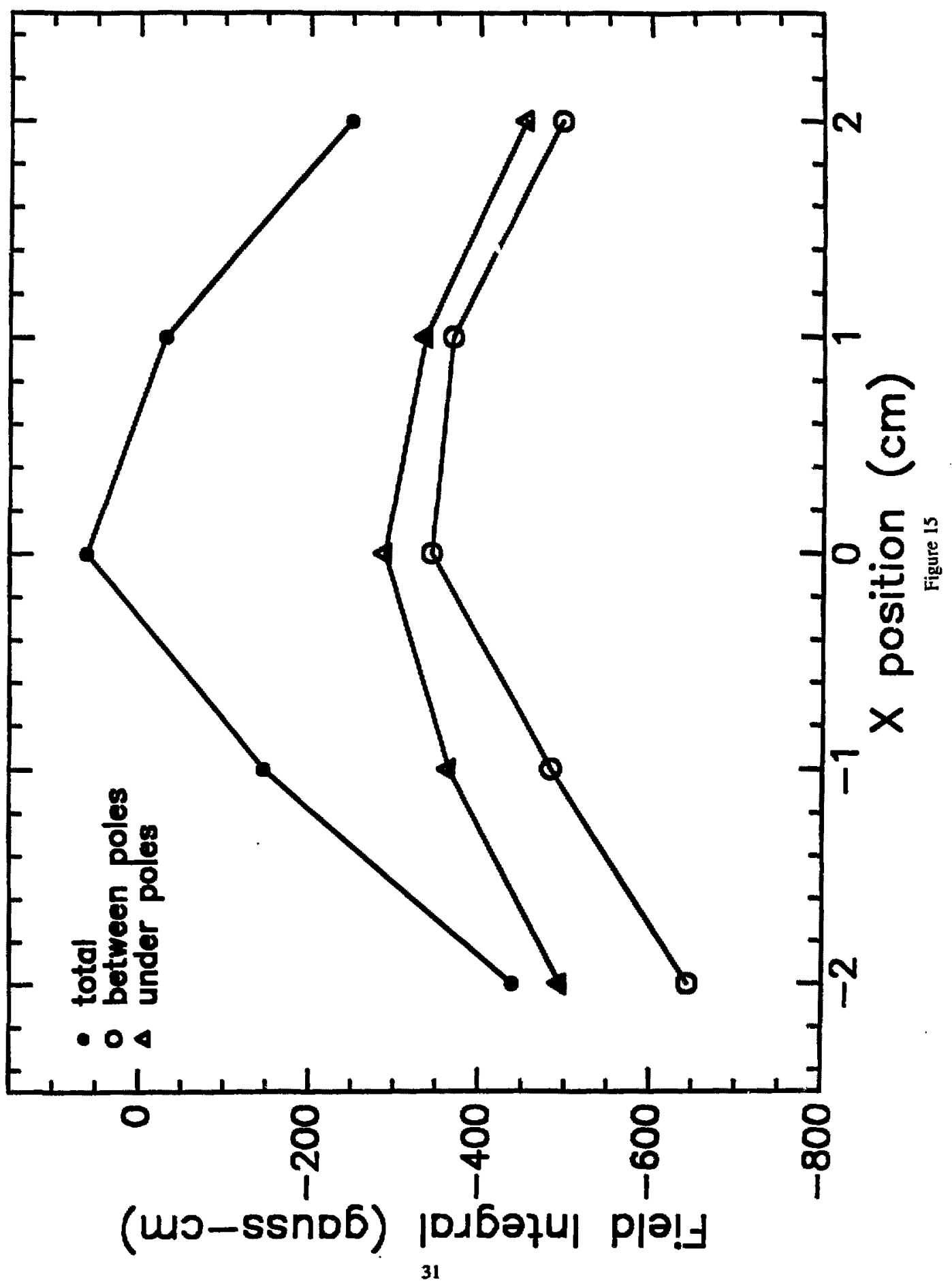




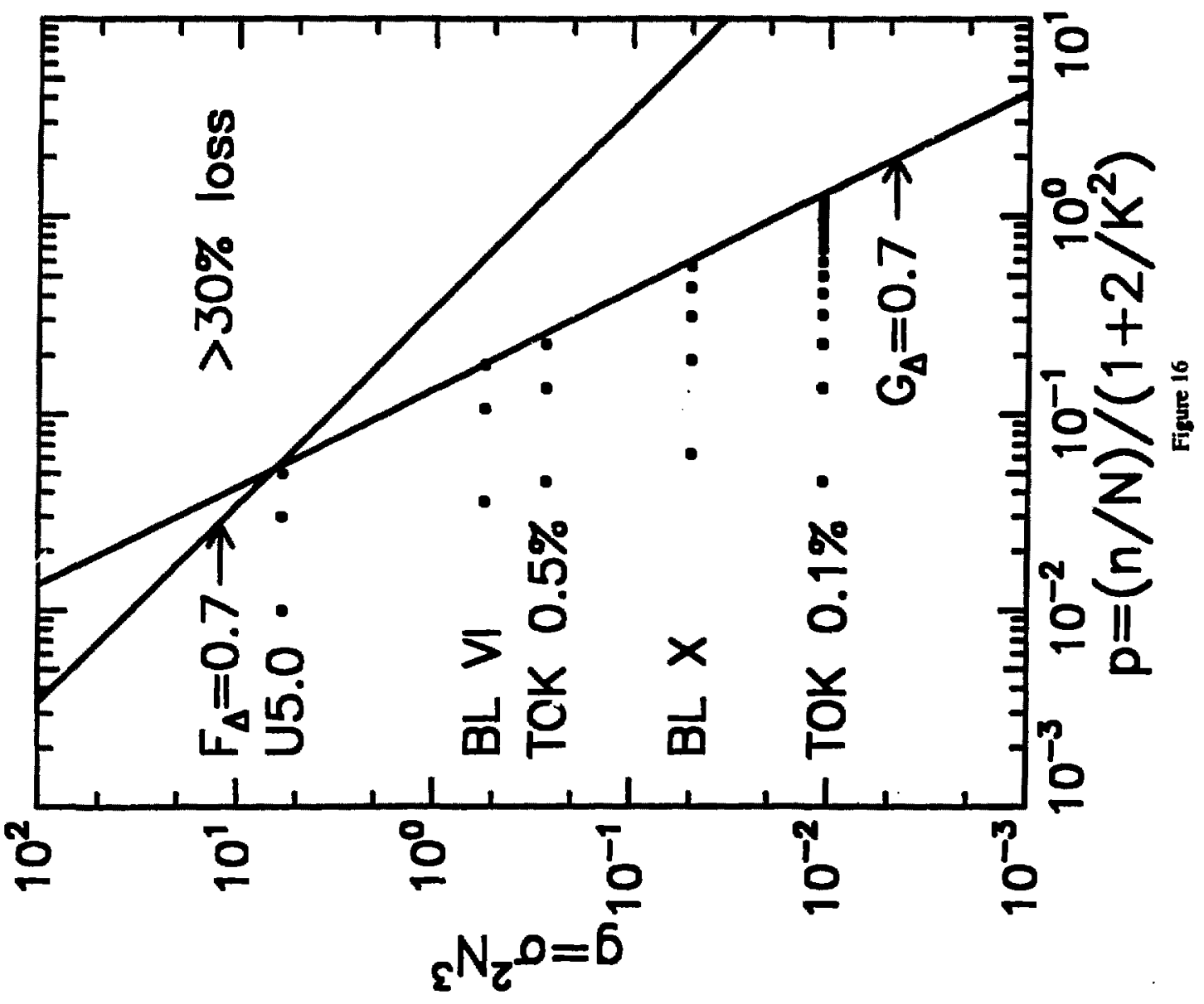

